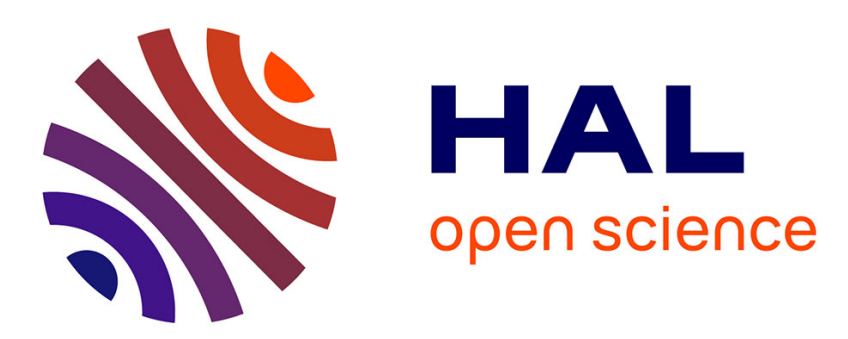

\title{
On the Contribution of Adhesion and Friction in Planning Dexterous in-Hand Micromanipulation
}

\author{
Jean Seon, Redwan Dahmouche, Michaël Gauthier
}

\section{To cite this version:}

Jean Seon, Redwan Dahmouche, Michaël Gauthier. On the Contribution of Adhesion and Friction in Planning Dexterous in-Hand Micromanipulation. Journal of Micro-Bio Robotics, 2017, 12 (1-4), pp.33 - 44. hal-02868183

\section{HAL Id: hal-02868183 \\ https://hal.science/hal-02868183}

Submitted on 15 Jun 2020

HAL is a multi-disciplinary open access archive for the deposit and dissemination of scientific research documents, whether they are published or not. The documents may come from teaching and research institutions in France or abroad, or from public or private research centers.
L'archive ouverte pluridisciplinaire HAL, est destinée au dépôt et à la diffusion de documents scientifiques de niveau recherche, publiés ou non, émanant des établissements d'enseignement et de recherche français ou étrangers, des laboratoires publics ou privés. 


\title{
On the Contribution of Adhesion and Friction in Planning Dexterous in-Hand Micromanipulation
}

\author{
Jean-Antoine Seon · Redwan Dahmouche · Michaël Gauthier
}

Received: date / Accepted: date

\begin{abstract}
Micromanipulation and micro-assembly techniques play a key role in the development of new integrated smart systems with applications in strategic fields such as transport, telecommunication, health and defense. However, existing micro-handling solutions lack speed, flexibility and autonomy, which represents an obstacle to the development of these technologies. In previous works, we developed and validated new micro-manipulation techniques using dexterous multi-fingered micro-hands as well as a trajectory planner to automate the manipulation process. The present paper focuses on studying the influence of friction coefficient and adhesion forces at the micro-scale on the fingers' trajectories.
\end{abstract}

Keywords Micromanipulation - Dexterous in-hand manipulation · Trajectory planning $\cdot$ Adhesion forces

\section{Introduction}

At the macro-scale, industrial robots are able to perform several tasks automatically: manipulating, assembling, welding, etc. They are typically composed of a robotic structure (serial or parallel) allowing several degrees of freedom (DoF) positioning of a tool. In manipulation and assembly tasks, the tool is usually a simple gripper whose design is adapted to the manipulated objects. In both industrial applications and humanoid robots, dexterous hands are currently developed to make the robots able to grasp objects of various shapes with the same gripper (or hand) [1] [2] [3].

Contrary to the macro-scale, micro-manipulations are usually limited to simple pick and place operations [4] [5]. Accurate multi-axes rotational positioning of micro-objects is

The authors are with FEMTO-ST Institute, Univ. Bourgogne FrancheComté, CNRS, 24 rue Savary, 25000 Besançon, France.

E-mail: ja.seon@femto-st.fr, redwan.dahmouche@femto-st.fr, michael.gauthier@femto-st.fr particularly difficult to obtain, which limits the micro-assembly possibilities [6] [7] [8]. Since it is not trivial to obtain multiDoF arms able to perform rotations at the micro-scale, a possible solution is to use a basic arm (translation micro-stages for instance) and a dexterous hand composed of translating fingers [9] [10].

Another characteristic related to manipulation at the microscale is the presence of surface forces which predominate over gravitational and inertial forces. For more than a decade, these sticky forces (Van der Waals, electrostatic and capillarity forces) have been considered as perturbation forces by the micro-robotics community, which has tried to get rid of them. Indeed, the manipulated micro-objects usually stick to the substrate during the grasping (a high pull-off force has to be applied) and then stick to the gripper during the release [11]. As these forces make the automation difficult, micro-manipulation operations are often done manually [12]. This solution is usually not satisfactory as the number of micro-systems units to produce is usually huge.

Contrary to various approaches that try to minimize this effect in order to fall back to dexterous manipulation at the macro-scale [13] [14], we propose to take advantage of these adhesion forces. Indeed, these forces can contribute to the stability of the object during the manipulation and allows to generated original trajectories [15]. Previous experiments have shown that original grasping configuration using only one finger can be sufficient to have a stable "grasp" (Fig.1).

In a previous work we have shown through simulation and experimentation that the presence of adhesion forces have a significant impact on the generated finger trajectories for in-hand manipulation. This paper is an extension of a previously published conference paper [16]. The main contribution of this paper is the study of adhesion forces and friction coefficient impact on dexterous micro-manipulation and on the optimal fingers trajectories planned with our algorithm. 


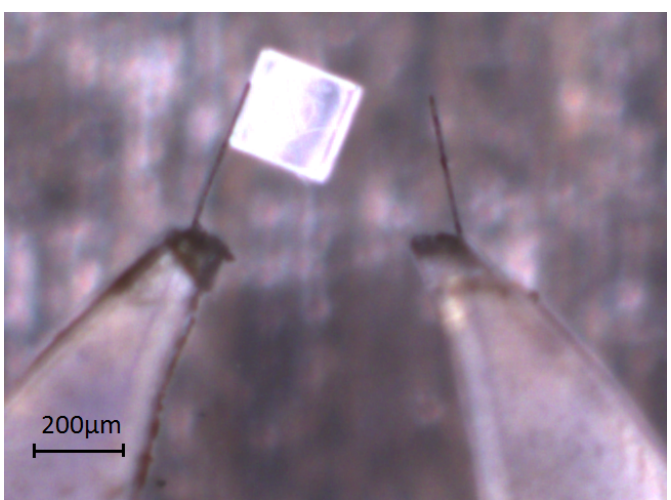

Fig. 1: Illustration of the adhesion effect: a non-intuitive grasp leads to a stable grasp.

The next section gives an overview of the in-hand micromanipulation problem while section 3 presents the main outcomes of our previous works on trajectory planning. Section 4 evaluates the impact of adhesion forces on the optimal trajectories. Then, section 5 presents the influence of friction during the trajectory planning for multi-fingered micromanipulation. In section 4 and 5, various trajectories are presented to illustrate the influence of each parameters and their coupling. Finally section 6 explains how to deal with uncertainties on these parameters to generate reliable trajectories.

\section{Dexterous in-Hand Manipulation: Micro vs Macro}

In-hand manipulation refers to the ability to translate and rotate an object using multiple fingers. At the macro-scale, in-hand manipulations can be achieved in different ways: rolling [17], sliding [18] and finger gaiting [19]. However, in-hand micromanipulations performed with controlled slip are very complicated because of the uncertain contact properties and the lack of accurate force sensing at this scale. Thus, it seems more reasonable to use only rolling and finger gaiting in dexterous micro-manipulation.

Furthermore, macro-manipulation is focused on designing anthropomorphic hands which have to be very versatile in order to quickly grasp unknown objects (usually in the context of humanoid robotics). Since dexterous micromanipulation aims at performing micro-assembly, the shapes of the manipulated object are assumed to be known and their surface properties roughly estimated.

Moreover, as inertial forces are negligible compared to adhesion forces at the micro-scale, it is possible to consider the micro-manipulation process under a quasi-static assumption where dynamics is neglected.

Finally, it is well admitted by the micro-manipulation and micro-assembly community that adhesion forces exist and are predominant in the micro-scale. These forces help to stabilize the grasp but can also make the release difficult.

\section{Background}

This section presents the theoretical basics used to generate fingers' trajectories for dexterous micromanipulation. Our approach, as presented in [16] and summarized in Fig.2, is to generate several maps that represent all the equilibrium grasps and all the finger gaiting possibilities. Knowing that the manipulation is quasi-static at the small scales, the trajectory can be viewed as a succession of equilibrium grasps. Thus, once the maps are computed, navigating in this space allows to generate the finger trajectories. This step can be done using a graph search algorithm as each equilibrium grasp can be considered as a node in a graph. The remaining of this section is a reminder of our previous work [16].

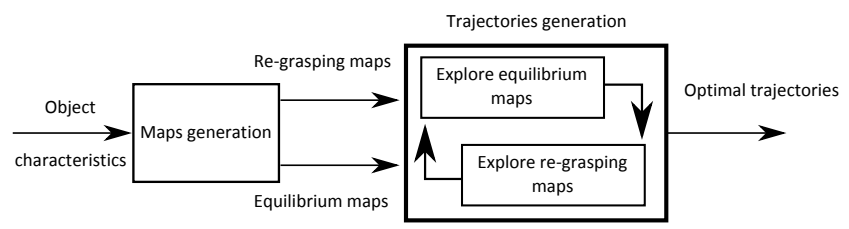

Fig. 2: Schematic representation of our approach used to generate optimal finger trajectories.

\subsection{Equilibrium and Re-grasping Maps}

Considering that the object surface is represented by a discrete regular sampling, the equilibrium and re-grasping maps (noted respectively $M_{k}$ and $D_{k}$ with $k$ the number of fingers used) are generated by testing all the grasping possibilities.

\subsubsection{Map Generation}

When generating the equilibrium map $M_{k}$, we test any grasp involving $k$ fingers to know if it is possible to keep the object at equilibrium. In the general case of six DoF manipulation, this means that all the grasping forces must be balanced:

$-w_{\text {ext }}=G \cdot f_{c}=\sum_{i=1}^{k} w_{c_{i}}$

where $w_{\text {ext }}$ is the external wrench (force and torque) applied on the object (the insertion force during an assembly for instance), the matrix $G \in \Re^{6 \times 3 k}$ is called the grasp matrix [20] which depends on the contacts positions, $f_{c} \in \mathfrak{R}^{3 k}$ is the vector containing the $k$ grasping forces and $w_{c_{i}} \in \mathfrak{R}^{6}$ is the $i^{t h}$ grasping wrench.

Thus, finding a stable grasps is equivalent to finding a set of forces $f_{c}$ verifying Eq. 1 and a non-slippage constraint. At the microscale, the non-slippage constraint can be expressed 
using the Coulomb law where the adhesion forces are taken into account [15]:

$\sqrt{f_{t_{1}}^{2}+f_{t_{2}}^{2}} \leq \mu\left(f_{n}+f_{p o}\right)$

where $f_{t_{1}}$ and $f_{t_{2}}$ are tangential components of the force, $f_{n}$ is the normal component parameter, $f_{p o}$ is the adhesion force amplitude and $\mu$ is the friction coefficient.

The adhesion allows to apply both positive and negative grasping forces. This means that it is possible to push and pull the object as long as the force lies in the modified friction cone. Considering this contact model, it is possible to express the wrench applied by the $i^{t h}$ finger, $w_{c_{i}}$, as a linear combination of $j$ wrenches that approximate the friction cone:

$$
\left\{\begin{array}{c}
w_{c_{i}}=\sum_{j} \alpha_{i, j} \cdot w_{l_{j}, i}+\beta_{i} \cdot w_{p o_{i}} \\
\alpha_{i, j} \geq 0 \\
\beta_{i} \geq 0
\end{array}\right.
$$

where $w_{l_{j}, i}$ is one wrench that approximate the $i^{\text {th }}$ friction cone, $w_{p o_{i}}$ the wrench induced by adhesion forces, and $\alpha_{i, j}$ and $\beta_{i}$ coefficients which must be positive to stay inside the friction cone. Thus, it is possible to rewrite equation (1) as follows:

$-w_{\text {ext }}=\sum_{i=1}^{k} w_{c_{i}}=\sum_{i=1}^{k}\left(\sum_{j} \alpha_{i, j} \cdot w_{l_{j}, i}+\beta_{i} \cdot w_{p o_{i}}\right)$

Thus, finding a stable grasp is equivalent to finding a set of $\left(\alpha_{i, j}, \beta_{i}\right)$ positive (not all of them null) verifying the previous equation. This step can be achieved by testing if the convex hull formed by the wrenches $\left(w_{l_{j}, i}\right.$ and $\left.w_{p o_{i}}\right)$ contains the external wrench [21]. Indeed, the convex hull represents the set of forces that it is possible to apply on the object with a given grasping configuration. So a necessary and sufficient condition to obtain a stable grasp is that the external force is included in this set. Then, the equilibrium maps can be formalized as follows:

$$
\begin{aligned}
M_{k}= & \left\{\left(c_{1}, . ., c_{k}\right) \in \mathfrak{R}^{(3 \times k)}\right. \\
& \left.-w_{\text {ext }} \in \operatorname{Convhull}\left(w_{l_{1}, 1}, \ldots, w_{l_{j}, k}\right)\right\}
\end{aligned}
$$

where $c_{k}$ is a vector containing the contact coordinates on the object surface and Convhull $\left(w_{l_{1}, 1}, \ldots, w_{l_{j}, k}\right)$ represents the convex hull.

Figure 3 depicts an example of a planar grasp and its corresponding convex hull (no external forces are applied and no adhesion is considered). In this planar case, each wrench is a vector containing three coordinates: the forces acting on the $x$ and $y$ axis and the moment acting on the $z$ axis.
Since adhesion is not considered in this example, each friction cone can be decomposed in two vectors representing the projection of the cone on the plan. Thus, the convex hull is formed by six wrenches. It can be seen that the origin of the wrench space is included in the hull. This grasp is stable and included in the $M_{3}$ map.
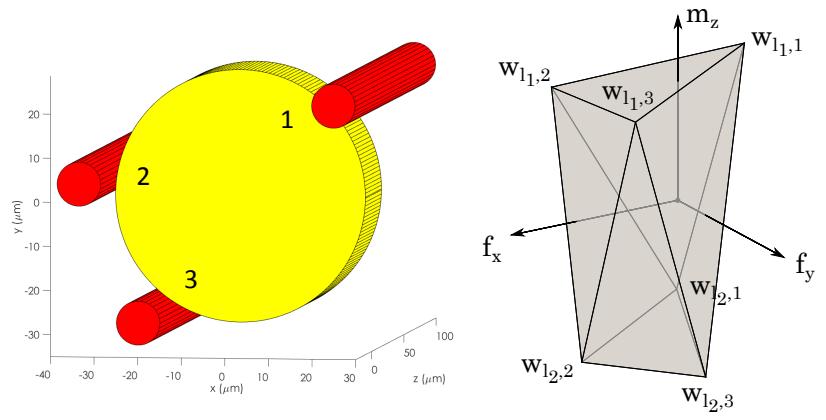

Fig. 3: Illustration of a three-finger planar grasp and its corresponding convex hull. The origin is included in the hull meaning that this grasp is stable.

The re-grasping maps are obtained similarly. When a finger is detached from the object, the remaining fingers must resist the external wrench applied by the pull-off of the removed finger $w_{p o_{r}}$ :

$-w_{\text {ext }}-w_{p o_{r}}=G \cdot f_{c}=\sum_{i=1}^{k-1} w_{c_{i}}$

Thus, for each $M_{k}(k>1)$ it is possible to define a corresponding re-grasping map $D_{k}$ showing whether the grasp remains stable while removing a finger or not:

$$
\begin{aligned}
D_{k}= & \left\{\left(c_{1}, . ., c_{k}\right) \in \mathfrak{R}^{(3 \times k)} \mid\left(c_{1}, . ., c_{k-1}\right) \in M_{k-1},\right. \\
& \left.-w_{p o, k} \in \text { Convhull }\left(w_{l_{1}, 1}, \ldots, w_{l_{k}, k-1}\right)\right\}
\end{aligned}
$$

\subsubsection{Example of Maps}

For any object shape, it is possible to create the various $M$ and $D$ maps. Indeed, considering that the object shape is represented by an arbitrary number of sample points (distributed homogeneously), the maps are obtained by testing every combination of contact point. Moreover, in the remaining of the paper, we consider only planar objects.

Figure 4 gives a representation of the $M_{2}$ set in the case of a planar circle without external perturbations. For simplicity of representation, the contact position of each finger is represented using the curvilinear abscissa. More precisely, the curvilinear abscissa of each finger represents the 
arc length between the contact point and an arbitrary origin. The depicted areas represent the equilibrium configurations in the case of a two-finger grasp without any adhesion forces. Obviously such types of sets are symmetric since contact point permutation represents the same grasp. Physically, the strip width which represents the stable grasp is proportional to the friction cone's aperture.

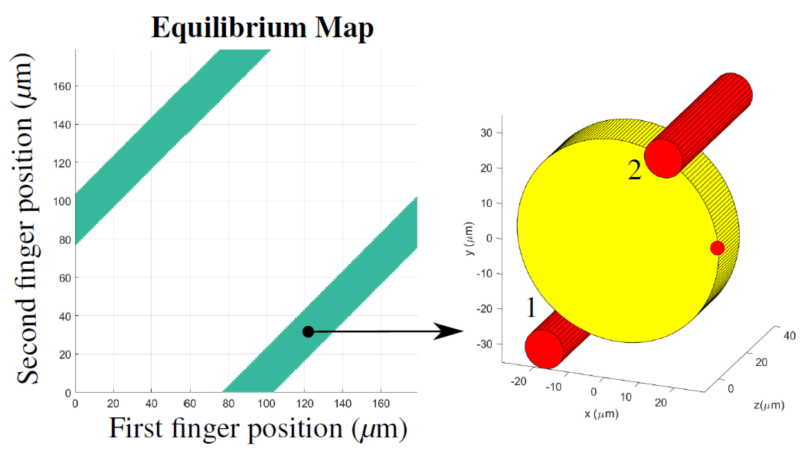

Fig. 4: Representation of the set $M_{2}$ (equilibrium map) for a circle, without considering adhesion forces (non-sticky behavior, $\forall i, w_{p o_{i}}=0$ ). The finger position is represented using the curvilinear abscissa of the contact point (the red spot indicates the curvilinear abscissa origin).

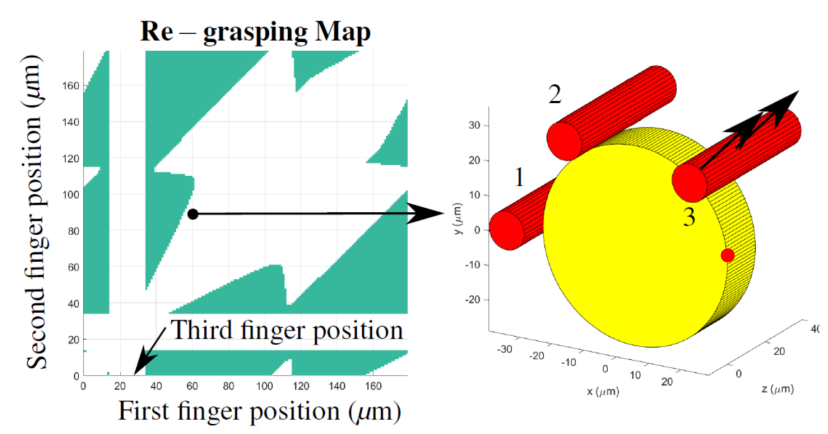

Fig. 5: Representation of the set $D_{3}$ (re-grasping map) for an assigned location of the third finger (curvilinear abscissa = $30 \mu \mathrm{m}$ ) considering adhesion (sticky behavior, $\forall i, w_{p o_{i}} \neq 0$ ). The finger position is represented using the curvilinear abscissa of the contact point (the red spot indicates the curvilinear abscissa origin).

Figure 5 (left) gives an example of a subset of the $D_{3}$ map in the case of a planar circle.. Considering that the position of the removed finger is not varying (it is always positioned at $30 \mu \mathrm{m}$ ), this map represents all the grasping configuration that guarantee that the third finger can be removed. The grasp depicted in Fig.5 (right) is stable with the three fingers and it is also stable with only finger 1 and 2 (thanks to the adhesion). However, this map shows that this config- uration leads to instability when removing the third finger. Obviously, this subset of $D_{3}$ is varying with the position of the removed finger. Thus $D_{3}$ is the union of such subsets.

\subsection{Generating trajectories}

Considering any planar motion, it is required to generate reliable trajectories able to perform the desired displacement. Object translations are easily generated by translating all the fingers in the same direction and on the same distance. However, rotations induce a displacement of the contact point and thus a displacement in the $M$ map. Consequently, it is possible to plan any rotational motion by navigating inside and between the various maps. More precisely, this step is done using a graph search algorithm that allows to explore the graph formed by the stable grasp.

From an initial grasp with $k$ fingers, the $M_{k}$ map is explored to generate the rotational motion. The rolling without sliding strategy constrains the search in this map. Indeed, if the fingers have the same radius, it is required that they move in the same direction on the object surface and on the same distance. Then, once a limit is reached in the $M_{k}$ map, for example the friction cone limit, the grasp must be reconfigured by removing or placing a finger. Placing a finger is just switching from $M_{k}$ to $M_{k+1}$ after checking the feasibility but removing a finger require to look for an available node in $D_{k}$. These actions are repeated until the whole rotation is completed.

In our previous works [15], we chose to use the $A^{*}$ algorithm to perform this graph search. This algorithm requires to define a cost function for each possible action and to define a heuristic. We chose the cost functions in such a way that they estimate the distance traveled by the fingers. For example, when a finger is added, the cost function estimates the distance between the current position of the finger and its goal position. Additionally, we chose the remaining rotation (expressed as the rolling distance) as the heuristic. This means that our algorithm is designed to generate trajectories that are optimal with regards to the traveled distance of the fingers.

\section{Impact of Adhesion Forces}

The algorithm presented above allows to generate trajectories for dexterous planar micromanipulation and takes into account the adhesion forces. Since it is not trivial to estimate the interaction forces at the microscale, it is interesting to study the influence of this parameter on the trajectories and, more particularly, how the adhesion and its value impacts the manipulation behavior and its robustness. In this section, when adhesion forces are considered, we assume 
that the strength of adhesion $f_{p o}$ is the same for each finger and that the weight is negligible compared to the adhesion.

\subsection{Impact of the Presence of Adhesion Forces}

First of all, let us show the effect of adhesion forces on the grasp stability maps $(D$ and $M$ ) used for the trajectory generation. For instance, we consider the $M_{2}$ map described in Fig. 6 to show the impact of adhesion on the two-finger equilibrium map. Note that this map corresponds to the object depicted in Fig.9.

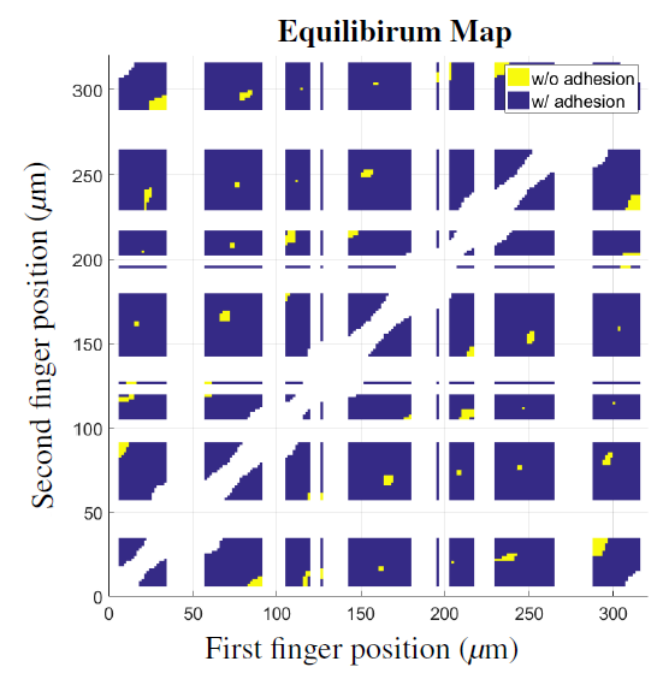

Fig. 6: Representation of the set $M_{2}$ (two-finger equilibrium map) with and without adhesion for an arbitrary shaped object. The finger position is represented using the curvilinear abscissa of the contact point.

Figure 6 shows the stabilizing effect of adhesion on an arbitrarily shaped object. In this example, we used a friction coefficient on the object surface of $\mu=0.3$, an empirically chosen adhesion force of $1 \mu \mathrm{N}$, a neglected external wrench and fingers with a radius of $7 \mu \mathrm{m}$. The blue and yellow areas on the map represent the stable grasps using two fingers in the presence and absence of adhesion forces respectively. As predicted, the stable area is significantly bigger when adhesion is present. This means that adhesion contributes to stabilize the grasp which offers more possibilities to manipulate the object.

Figure 7 shows the admissible grasping configuration when the third finger has to be removed (the position of the removed finger is predefined). It can be seen that without adhesion all the stable grasps with two fingers (yellow areas in Fig.6) are also admissible for finger gaiting (no pull-off disturbing forces). We can also note that, even if the two fingers grasps are pretty stable (blue areas on Fig.6), getting to

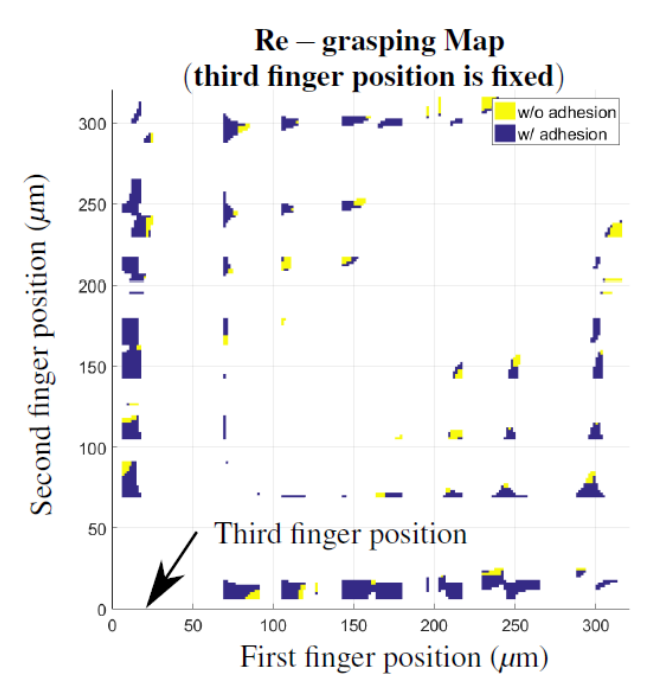

Fig. 7: Representation of the set $D_{3}$ (re-grasping map) with and without adhesion for an arbitrarily shaped object and a fixed position of the removed finger. The finger position is represented using the curvilinear abscissa of the contact point.

these grasps from a three fingers grasp is constrained (blue areas in Fig. 7 considerably shrink).

\subsection{Impact of the Strength of Adhesion Forces}

The presence of adhesion forces has a strong impact on the maps but it is important to estimate the influence of its strength. We consider the same parameters as in the previous example (neglected external wrench, friction coefficient of 0.3 and fingers radius of $7 \mu \mathrm{m}$ ) but with different values of adhesion forces.

Figure 8, which shows the number of stable grasps as a function of the adhesion force, highlights the fact that the adhesion value has no impact on the number of stable grasps. Indeed, whether the adhesion is $0.5 \mu \mathrm{N}, 5 \mu \mathrm{N}$ or $10 \mu \mathrm{N}$, the number of stable grasp remains constant and the set of stable grasps is unchanged. This means that, as long as the adhesion value is higher than the neglected external wrenches, this value will have literally no impact on the stable grasp and the generated trajectories.

\subsection{Impact of Adhesion on the Generated Trajectories}

As the maps used to generate the trajectories are impacted by the presence of adhesion, it is interesting to see its effect on the trajectories themselves. Let us consider the three-finger manipulation case. The manipulation space is then represented by $M_{2}, M_{3}$ for grasping and rolling and $D_{2}, D_{3}$ for re-grasping and finger gaiting. The following results have 


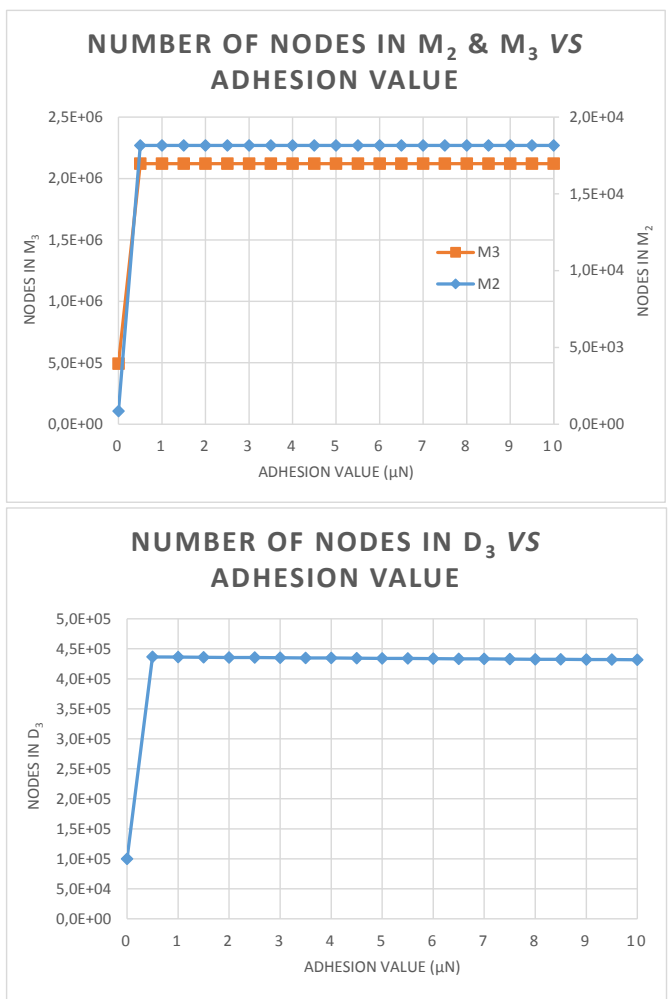

Fig. 8: Charts representing the evolution of the number of elements in $M_{2}$ and $M_{3}$ (equilibrium map) and $D_{3}$ (re-grasping map) for various adhesion values (0 to $10 \mu \mathrm{N})$.

been generated using the same parameters as before and for two values of adhesion: null and $1.5 \mu \mathrm{N}$. Note that this value of adhesion is set empirically since it has no impact on the stable grasping and re-grasping configurations as shown in the previous subsection.

Figure 9 illustrates the computed trajectory for a $147^{\circ}$ rotation without adhesion. The initial grasp depicted on Fig.9a used two fingers. From this configuration, the optimal path computed by the algorithm includes some rotation steps achieved by rolling the object on the fingers (represented in Fig.9b, d, $\mathrm{f}, \mathrm{h}$ ) and three re-grasping steps (represented in Fig.9c, e, g). However, in presence of adhesion (Fig.10), the optimal path does not use a finger gaiting step and the movement is performed in one rotation step. Thus, when adhesion exists, it allows to manipulate the object with less re-grasping steps and with original grasping configuration. In another paper [23], we have shown that the manipulation process is faster when the adhesion is used to manipulate the object.

Moreover, as the adhesion value has no impact on the various maps, it has also no impact on the trajectories. Thus, the trajectory generated with an adhesion force of $1.5 \mu \mathrm{N}$ is the same for any higher or smaller values of adhesion as long as external forces (including weigth) are negligible compared to the adhesion force.

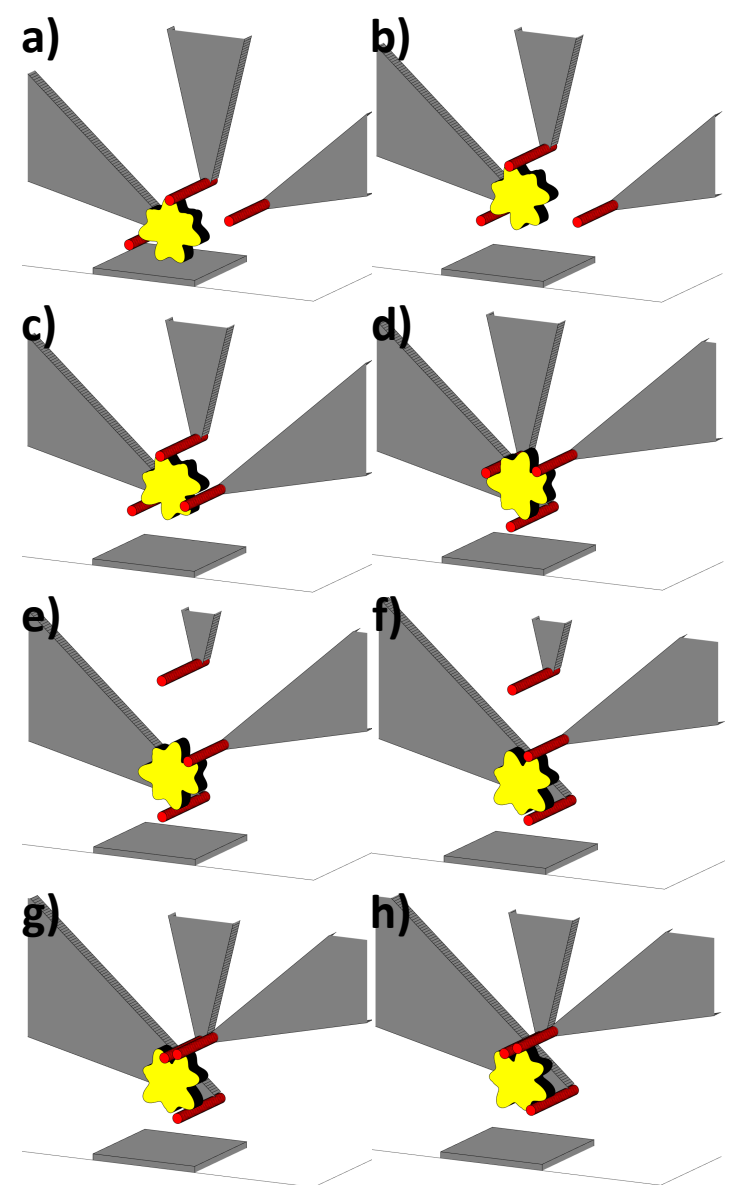

Fig. 9: Image sequence describing the trajectory generated for a $147^{\circ}$ rotation without adhesion. a) represents the initial grasp using three fingers while b), d), f) h) represent the rotation steps and c), e), g) the re-grasping steps.

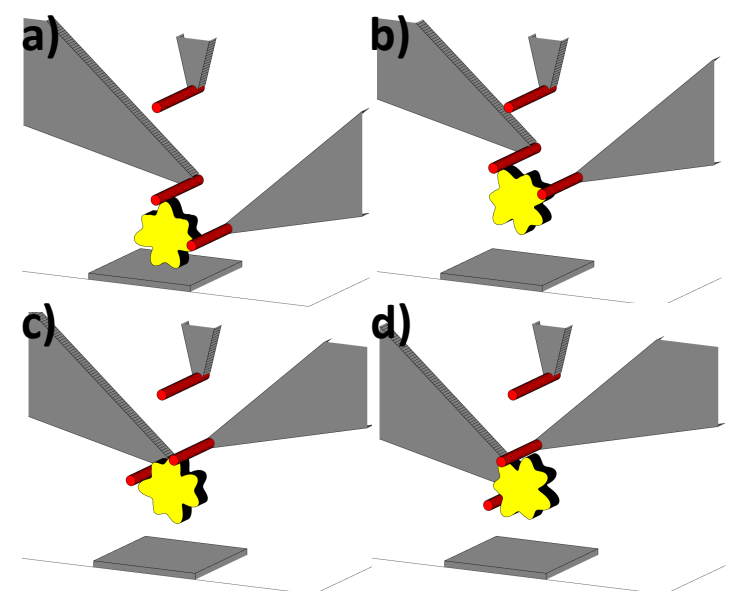

Fig. 10: Image sequence describing the trajectory generated for a $147^{\circ}$ rotation with adhesion. a) represents the initial grasp using two fingers while b), c), d) represent the rotation steps. 


\section{Impact of Friction Coefficient}

The friction coefficient is directly linked to the angle of the friction cone. Thus, it seems to have a significant impact on the trajectories and especially on the equilibrium maps. This section aims to evaluate its influence on the grasp stability and study the coupling between friction and adhesion. Similarly to the previous section, we assume that the value of adhesion is the same for each finger and that the weight is negligible compared to the adhesion.

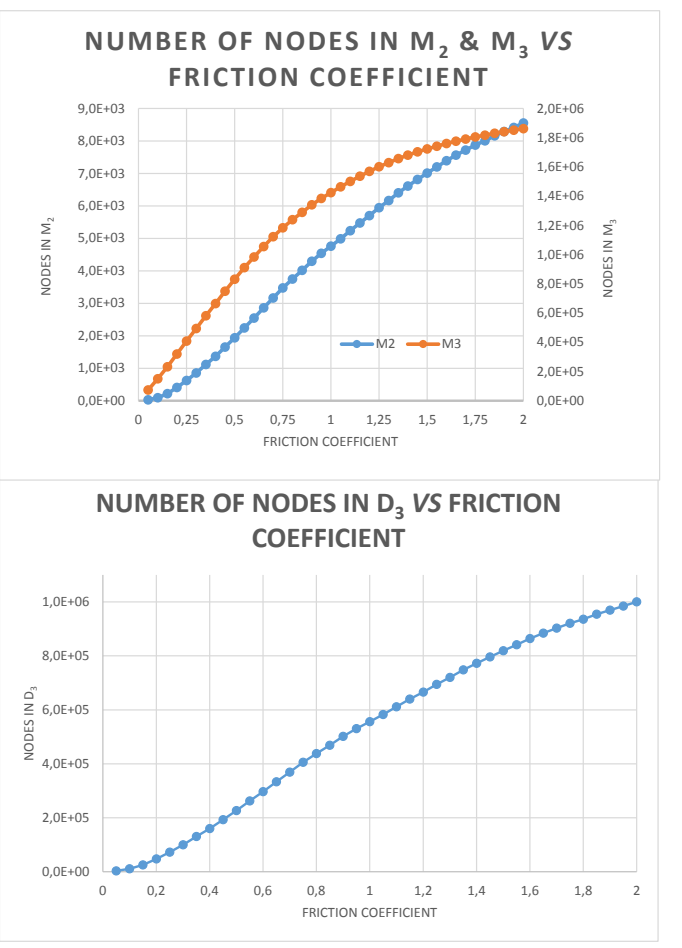

Fig. 11: Charts representing the evolution of the number of elements in $M_{2}$ and $M_{3}$ (equilibrium map) and $D_{3}$ (regrasping map) for various friction values ( 0.05 to 2 ).

\subsection{Friction Coefficient Impact on Grasp Stability}

First of all, we consider the case where the adhesion force is null. In order to evaluate the impact of the friction value, we generated maps with friction varying between 0.05 and 2 . Figure 11 shows that this parameter has an important impact on the number of two- and three-finger stable grasps ( $M_{2}$ and $M_{3}$ maps). Indeed, when adhesion is not present or not considered, the friction cone's aperture is the most influent parameter on the number of equilibrium and re-grasping configurations. It can be seen that the number of equilibrium grasps increases with the friction coefficient.
It is also interesting to note that the number of two- and three-finger grasps with a friction coefficient of 0.3 (represented in Fig.8) is always higher in presence of adhesion whatever the friction value is (represented in Fig.11). Concerning re-grasping nodes, we can note that it requires to have a friction value three times higher (Fig.11) to have the same number of nodes in $D_{3}$ when adhesion is present (Fig.8). Note also in this example that, even with a very high friction value, some grasping configuration will never be stable without adhesion.

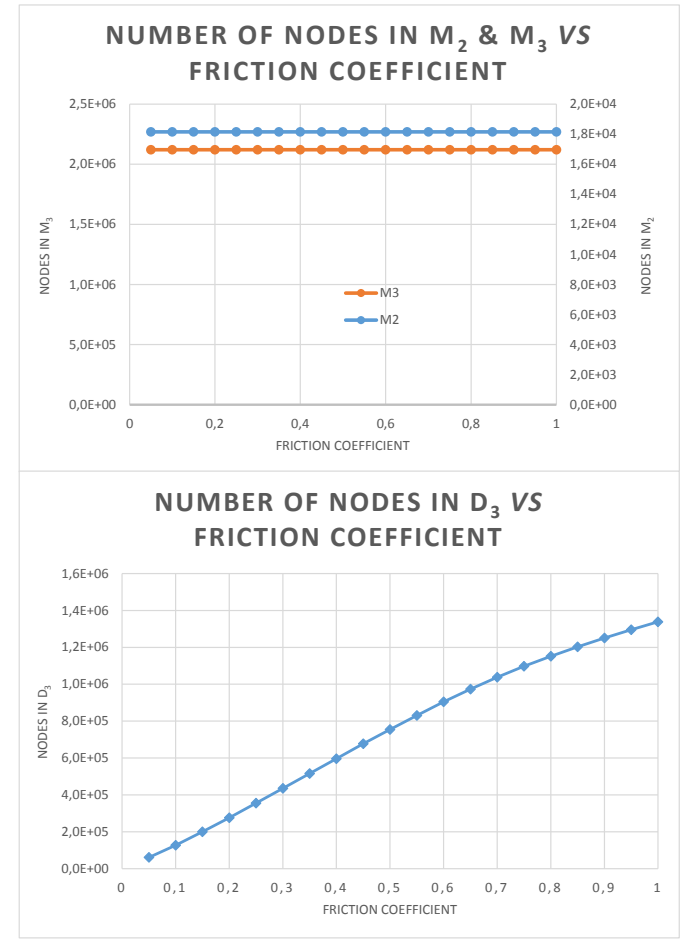

Fig. 12: Charts representing the evolution of the number of elements in $M_{2}$ and $M_{3}$ (equilibrium map) and $D_{3}$ (regrasping map) for various friction values (0.05 to 1) and an adhesion value of $1.5 \mu \mathrm{N}$.

\subsection{Impact of Friction with Adhesion}

Let us now consider the case of a varying friction in presence of adhesion (reminds that the value of adhesion has no impact so the results here are for any considerable adhesion value). The evolution of the number of nodes in $M_{2}, M_{3}$ and $D_{3}$ is illustrated in Fig.12 where it can be seen that friction has no impact on the stable grasp when adhesion is considered. Thus, for small rotations that can be performed with only two fingers and without finger gaiting, adhesion and friction have a very limited impact on the trajectories that can be generated. However, they have an impact on the num- 
ber of elements in $D_{3}$ (stable re-grasp configurations) which increases with the friction value. This means that higher friction coefficients increase the re-grasping possibilities when finger gaiting is required.

\subsection{Trajectories differences}

We have seen that the friction value has a more important impact on the stable grasps used to plan the trajectories than the adhesion forces' strength. To evaluate the influence of this parameter on the trajectories behavior, two cases are considered (presence or not of adhesion).

For the first case, we consider two values of friction coefficient: 0.3 and 1.5. Note that the friction coefficient of 1.5 seems unrealistic but it is used to highlight the impact of this parameter on the trajectories. Figure 13 represents the trajectory computed for a $180^{\circ}$ rotation using a friction coefficient of 0.3 . Rotating this object is a complex task which requires to perform five finger gaiting steps. It is interesting to note that the trajectory going from 0 to $144^{\circ}$ is very similar to the one depicted in Fig.9. Nevertheless, some contact positions are different (especially in Fig.13e and Fig.13f). This means that the optimal trajectory used to perform a rotation of $\theta^{\circ}$ is not necessarily included in the optimal trajectory able to perform a rotation of $\theta+\delta \theta^{\circ}$.

Figure 14 shows the computed trajectory using a friction coefficient of 1.5. It can be seen that increasing the friction value has a significant impact on the trajectory. Indeed, the number of finger gaiting steps is considerably reduced changing from five finger gaiting steps to only two.

In the second case where adhesion forces exist, the computed trajectories are depicted in Fig.15 for a 0.3 friction value and in Fig.16 for a 1.5 friction coefficient. It can be seen that, when adhesion is considered, the variation of the friction coefficient has a very limited impact. Indeed for these two trajectories, the number of re-grasping steps is the same (two steps illustrated in Fig.15c,e and Fig.16c,e). The only difference between these two trajectories is the contact position of the placed and removed finger during the re-grasping (highlighted by red circles). That was quite predictable as the friction only impacts the re-grasping maps when adhesion is used.

\section{Dealing with uncertainties}

In the previous sections, we have shown that friction and adhesion have different impacts on trajectory planning. In micromanipulation these parameters (adhesion and friction) are usually not precisely estimated. So the question is: how could the uncertainties on the friction coefficient and on the adhesion value be taken into account to generate a reliable manipulation process?

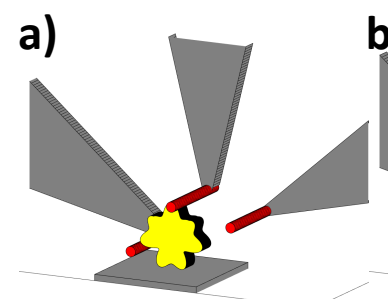

b)
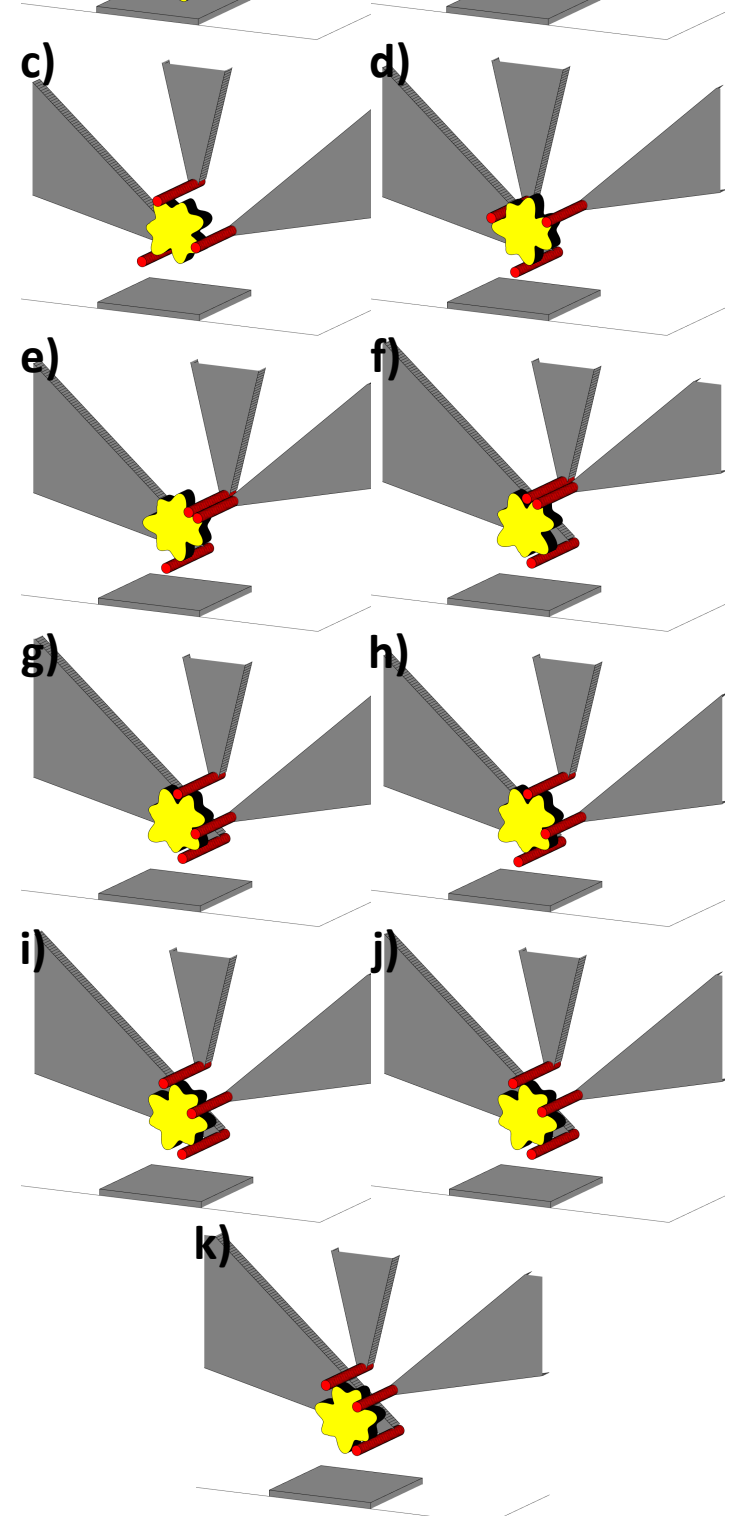

Fig. 13: Image sequence describing the trajectory generated for a $180^{\circ}$ rotation with a friction value of 0.3 and no adhesion. a) represents the initial grasp using two fingers while b), d), f), i), k) represent the rotation steps and c), e), g), h), j) the re-grasping steps.

Concerning the adhesion, we have shown that its value has no impact on trajectory planning as long as adhesion forces predominate over weight. Indeed, if the weight or any other forces acting on the object are of the same order as the adhesion forces, the maps will change which will have an 

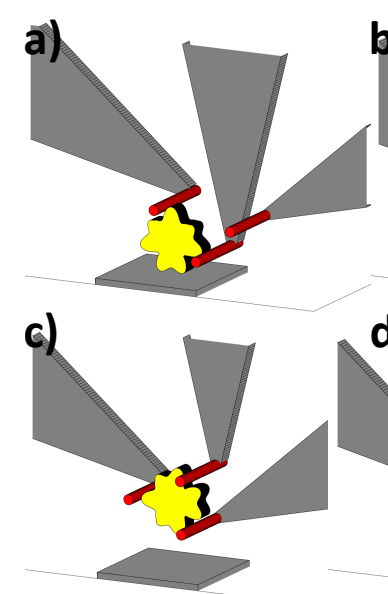

d)
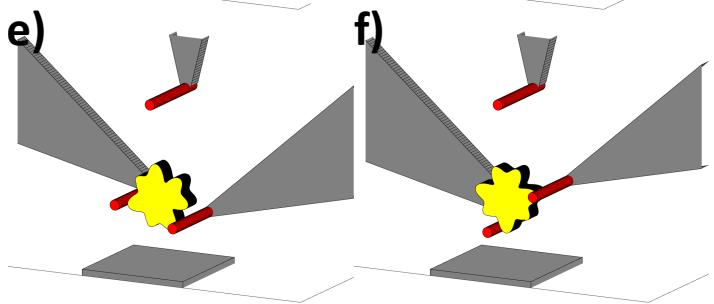

Fig. 14: Image sequence describing the trajectory generated for a $180^{\circ}$ rotation with a friction value of 1.5 and no adhesion. a) represents the initial grasp using two fingers while b), d), f) represent the rotation steps and c), e) the regrasping steps.

impact on the trajectories. More particularly, it is possible to generate trajectories where gravity and adhesion are of the same order. However, if the estimation of the adhesion is not accurate then the trajectories will not be robust to uncertainties. Nonetheless, it is possible to make sure that the fingers become very sticky by using chemical treatment for surface functionalization [22]. Thus, the uncertainty on adhesion forces can be handled by making sure that they are the predominant forces.

Concerning the friction coefficient, it has different impacts depending on whether the manipulation takes advantage of adhesion or not. If the manipulation does not take advantage of adhesion forces, then a safe way to deal with uncertainties is to generate trajectories using an underestimated friction coefficient. Finally, while generating trajectories considering adhesion, the friction value has only an impact on the re-grasping nodes. On the previous example in Section 4, it can be seen that high amplitude rotations are generated without finger gaiting. For example, with fingers of $7 \mu \mathrm{m}$ radius and a predominant adhesion force, it is possible to rotate the object presented in this paper over $180^{\circ}$ without re-grasping. Note that in other papers [15], [16], [23], we have experimentally shown that it was possible to rotate different objects over $140-160^{\circ}$ without finger gaiting. Thus, even if the friction value is underestimated, this
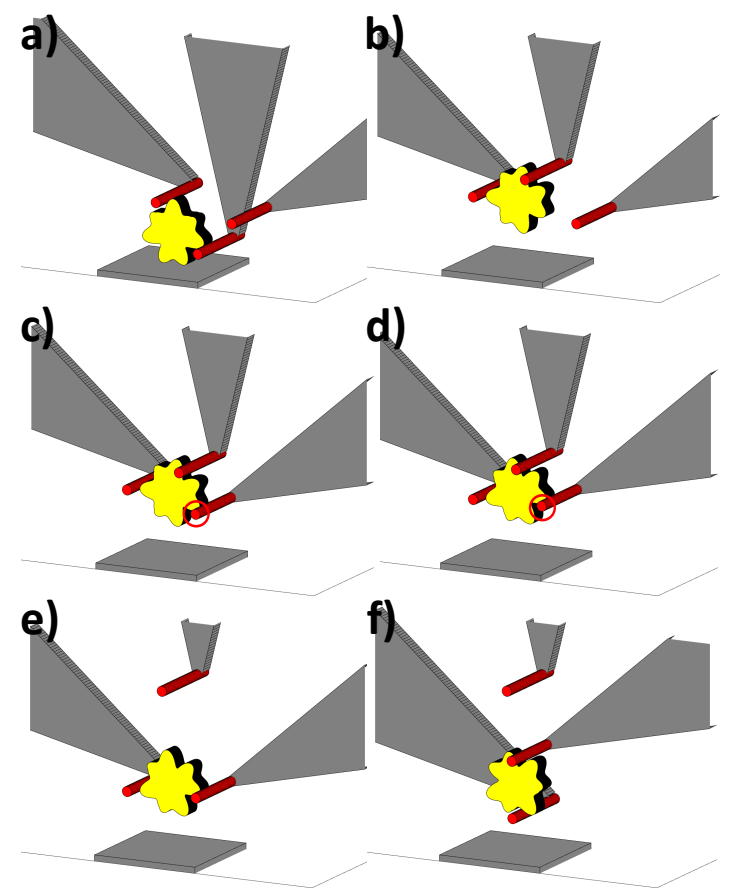

Fig. 15: Image sequence describing the trajectory generated for a $220^{\circ}$ rotation with a friction value of 0.3 and adhesion. a) represents the initial grasp using two fingers while b), d), f) represent the rotation steps and c), e) the re-grasping steps.
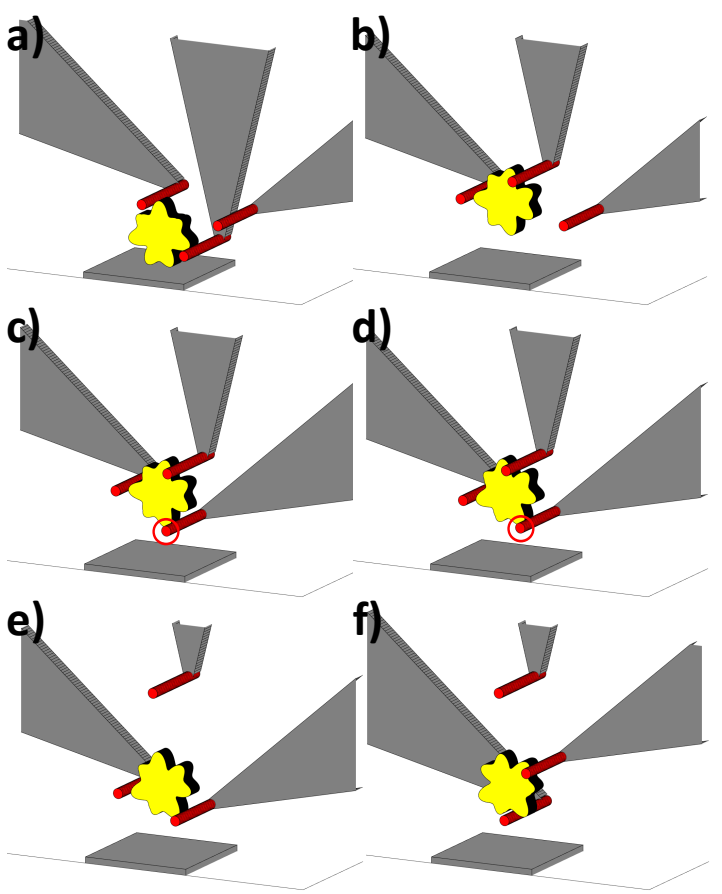

Fig. 16: Image sequence describing the trajectory generated for a $220^{\circ}$ rotation with a friction value of 1.5 and adhesion. a) represents the initial grasp using two fingers while b), d), f) represent the rotation steps and c), e) the re-grasping steps. 
will not change the trajectories so much. However, it is important to never overestimate the friction coefficient because this can lead to re-grasping failure where the finger we try to detach will remain stuck to the object.

\section{Conclusion}

In this paper we have investigated the impact of adhesion forces and friction coefficient on trajectory planning for multifingered micromanipulation. We have highlighted the fact that knowing precisely the adhesion's strength, which is investigated by many micro-roboticists, is not required for planning micromanipulation. Indeed, it is sufficient that these forces are dominant. Also, we have shown that friction plays an important role for planning manipulation when adhesion is not present but its impact is less visible once adhesion is present. Thus, knowing accurately the friction coefficient is also not required for planning dexterous micromanipulation.

Our approach has also shown that the presence of adhesion has a significant impact on trajectory planning and allows to generate original manipulation processes. The trajectories generated with this algorithm have not been validated experimentally in this paper. However we have validated a proof of concept at the millimeter scale with preponderant adhesion forces [23].

The next step of this work will involve the validation of the trajectories at the micrometer scale with a three fingered micro-hand developed in our laboratory. In addition, improving the trajectory planner by adding learning to increase its adaptability is planned. Finally, extending these results into three dimensional micromanipulation is also currently investigated.

Acknowledgements This work was supported by ACTION, the French ANR Labex no. "ANR-11-LABX-01-01", by the Equipex ROBOTEX project (contract "ANR-10-EQPX-44-01") and by the Conseil Régional de Franche-Comté.

\section{References}

1. Ma, R.R., Dollar, A.M.: On dexterity and dexterous manipulation. In: 15th International Conference on Advanced Robotics, pp. 1-7. IEEE (2011)

2. Koganezawa, K., Ito, A.: Artificial hand with stiffness adjuster. In: International Conference on Intelligent Robots and Systems, pp. 21-27. IEEE (2014)

3. Saut, J.P.: Planification de mouvement pour la manipulation dextre d'objets rigides. Ph.D. thesis, Université Pierre et Marie CurieParis VI (2007)

4. Xie, H., Régnier, S.: Three-dimensional automated micromanipulation using a nanotip gripper with multi-feedback. Journal of Micromechanics and Microengineering 19(7), 075,009 (2009)

5. Chu, H.K., Mills, J.K., Cleghorn, W.L.: Dual-arm micromanipulation and handling of objects through visual images. In: International Conference on Mechatronics and Automation, pp. 813-818. IEEE (2012)
6. Rabenorosoa, K., Clevy, C., Lutz, P., Das, A.N., Murthy, R., Popa D.: Precise motion control of a piezoelectric microgripper for microspectrometer assembly. In: International Design Engineering Technical Conferences, pp. 769-776. American Society of Mechanical Engineers (2009)

7. Gauthier, M., Régnier, S.: Robotic micro-assembly. John Wiley \& Sons (2011)

8. Feddema, J.T., Xavier, P., Brown, R.: Micro-assembly planning with van der waals force. Journal of Micromechatronics 1(2), 139153 (2001)

9. Zhou, Q., Korhonen, P., Laitinen, J., Sjövall, S.: Automatic dextrous microhandling based on a 6-dof microgripper. Journal of Micromechatronics 3(3), 359-387 (2006)

10. Inoue, K., Tanikawa, T., Arai, T.: Micro hand with two rotational fingers and manipulation of small objects by teleoperation. In: International Symposium on Micro-NanoMechatronics and Human Science, pp. 97-102. IEEE (2008)

11. Chaillet, N., Régnier, S.: Microrobotics for micromanipulation. John Wiley \& Sons (2010)

12. Banerjee, A.G., Gupta, S.K.: Research in automated planning and control for micromanipulation. Transactions on Automation Science and Engineering 10(3), 485-495 (2013)

13. Savia, M., Koivo, H.N.: Contact micromanipulationsurvey of strategies. Transactions on Mechatronics 14(4), 504-514 (2009)

14. Dejeu, J., Bechelany, M., Rougeot, P., Philippe, L., Gauthier, M.: Adhesion control for micro-and nanomanipulation. ACS nano 5(6), 4648-4657 (2011)

15. Seon, J.A., Dahmouche, R., Brazey, B., et al.: Finger trajectory generation for planar dexterous micro-manipulation. In: International Conference on Robotics and Automation, pp. 392-398. IEEE (2016)

16. Seon, J.A., Dahmouche, R., Gauthier, M.: Planning trajectories for dexterous in-hand micro-manipulation using adhesion forces In: International Conference on Manipulation, Automation and Robotics at Small Scales, pp. 1-6. IEEE (2016)

17. Bicchi, A., Sorrentino, R.: Dexterous manipulation through rolling. In: International Conference on Robotics and Automation, vol. 1, pp. 452-457. IEEE (1995)

18. Brock, D.L.: Enhancing the dexterity of a robot hand using controlled slip. In: International Conference on Robotics and Automation, pp. 249-251. IEEE (1988)

19. Leveroni, S.R.: Grasp gaits for planar object manipulation. Ph.D. thesis, Massachusetts Institute of Technology (1997)

20. Bicchi, A.: On the closure properties of robotic grasping. The International Journal of Robotics Research 14(4), 319-334 (1995)

21. Ponce, J., Faverjon, B.: On computing three-finger force-closure grasps of polygonal objects. Transactions on Robotics and $\mathrm{Au}-$ tomation vol. 11, pp. 868-881. IEEE (1995)

22. Cot, A., Dejeu, J., Lakard, S., Rougeot, P., Gauthier, M.: Modeling of electrostatic forces induced by chemical surface functionalisation for microrobotics applications. In: International Conference on Intelligent Robots and Systems, pp. 2065-2070. IEEE (2013)

23. Seon, J.A., Dahmouche, R., Gauthier, M.: Enhancing dexterous micromanipulation strategies by exploiting adhesion forces. IEEE Transactions on Robotics [under review] (2016) 